\title{
Momentum distribution of confined bosons: Temperature dependence
}

\author{
J. Tempere, ${ }^{1}$ F. Brosens, ${ }^{1}$ L. F. Lemmens, ${ }^{2}$ and J. T. Devreese ${ }^{1,2, *}$ \\ ${ }^{1}$ Departement Natuurkunde, Universitaire Instelling Antwerpen, Universiteitsplein 1, B-2610 Antwerpen, Belgium \\ ${ }^{2}$ Rijksuniversitair Centrum Antwerpen, Universiteit Antwerpen, Groenenborgerlaan 171, B-2020 Antwerpen, Belgium
}

(Received 2 March 1998)

\begin{abstract}
The momentum distribution function of a parabolically confined gas of bosons with harmonic interparticle interactions is derived. In the Bose-Einstein condensation region, this momentum distribution substantially deviates from a Maxwell-Boltzmann distribution. It is argued that the determination of the temperature of the boson gas from the Bose-Einstein momentum distribution function is more appropriate than the currently used fitting to the high-momentum tail of the Maxwell-Boltzmann distribution. [S1050-2947(98)04710-6]
\end{abstract}

PACS number(s): 03.75.Fi, 32.80.Pj, 05.30.Jp

\section{INTRODUCTION}

The extreme low temperature required for the observation of Bose-Einstein condensation in alkali-metal-atom vapors is a major achievement of present day experimentation [1-3]. Unfortunately, the temperature itself is not directly observable. It has to be estimated from other, more accessible properties of the system, such as the velocity distribution of the vapor [4]. Because of the uncertainty relation between the inverse temperature and the energy of the system for a finite number of particles, it is not clear a priori how many particles are required for a reasonable thermodynamically defined concept of temperature. In the present paper we do not address this fundamental question but rather assume that the number of bosons in the system is sufficiently large to have a meaningful definition of the temperature.

Under this assumption, the experimental estimate of the temperature [4] is based on the hypothesis that the momentum distribution function is Maxwell-Boltzmann like, at least for sufficiently high momentum. This hypothesis deserves further investigation and a quantitative comparison to the momentum distribution of a parabolically confined BoseEinstein gas. To the best of our knowledge, the BoseEinstein momentum distribution in a parabolic confinement potential has not been documented before.

Using the methods for path integration developed in [57], we have been able to calculate the momentum distribution function of a gas of parabolically confined bosons with quadratic harmonic interparticle interactions. This calculation is presented in Sec. II. Other investigations using the same methodology [8] indicated that for weakly interacting systems, such as ${ }^{87} \mathrm{Rb}$ and ${ }^{31} \mathrm{Na}$, the interaction slightly influences the parameters involved in the model system and therefore have only a moderate influence on the momentum distribution.

In Sec. III the approximations are examined that lead to the Maxwellian momentum distribution function and a quantitative comparison is made between the temperature obtained in the Bose-Einstein distribution and the estimate of the temperature, obtained by fitting a Maxwell-Boltzmann

\footnotetext{
*Also at Technische Universiteit Eindhoven, NL 5600 MB Eindhoven, The Netherlands.
}

distribution to the tail of the measured velocity distribution. The conclusion from this analysis is that the estimate from the Maxwellian deviates substantially from the temperature of the Bose-Einstein distribution in the presence of a BoseEinstein condensate.

\section{MOMENTUM DISTRIBUTION FUNCTION OF PARABOLICALLY TRAPPED BOSONS}

The anisotropic one-body potential energy $V_{1}$ and the two-body potential energy $V_{2}$ of the model system consisting of $N$ bosons are given by

$$
\begin{gathered}
V_{1}=\frac{m}{2} \sum_{j=1}^{N}\left[\Omega_{x y}^{2}\left(x_{j}^{2}+y_{j}^{2}\right)+\Omega_{z}^{2} z_{j}^{2}\right], \\
V_{2}=\mp \frac{m \omega^{2}}{4} \sum_{j, l=1}^{N}\left(\mathbf{r}_{j}-\mathbf{r}_{l}\right)^{2},
\end{gathered}
$$

where $\mathbf{r}_{j}=\left(x_{j}, y_{j}, z_{j}\right)$ is the position of the $j$ th boson, $m$ is the mass of the bosons, and $\Omega_{x y}$ and $\Omega_{z}$ are the frequencies of the parabolic confinement in the $x y$ plane and along the $z$ axis, respectively. The model interparticle interaction is harmonic with frequency $\omega$ and can be either attractive $(-)$ or repulsive $(+)$. The renormalized frequencies in the $x y$ plane and along the $z$ axis will be denoted by $w_{x y}=\sqrt{\Omega_{x y}^{2} \mp N \omega^{2}}$ and $w_{z}=\sqrt{\Omega_{z}^{2} \mp N \omega^{2}}$, respectively. These frequencies are the eigenfrequencies of the internal degrees of freedom of the boson gas, whereas the confining frequencies $\Omega_{x y}, \Omega_{z}$ are the eigenfrequencies for the center-of-mass motion. The momentum distribution $n_{\text {Bose }}(\mathbf{p})$, giving the statistical mean number of bosons with a given momentum $\mathbf{p}$ in the canonical ensemble, can be written as

$$
\begin{aligned}
n_{\text {Bose }}(\mathbf{p}) & =\left\langle\sum_{j=1}^{N} \delta\left(\mathbf{p}-\mathbf{p}_{j}\right)\right\rangle \\
& =\int \frac{d \mathbf{q}}{(2 \pi)^{3}} e^{i \mathbf{q} \cdot \mathbf{p}}\left\langle\sum_{j=1}^{N} e^{-i \mathbf{q} \cdot \mathbf{p}_{j}}\right\rangle,
\end{aligned}
$$


where the angular brackets indicate quantum averages for boson statistics. These mean values can be calculated for a system at temperature $T=1 / k_{B} \beta$ using the path integral propagator in momentum space $\mathcal{K}\left(\mathbf{p}_{1}^{\prime \prime}, \ldots, \mathbf{p}_{N}^{\prime \prime} ; \beta \mid \mathbf{p}_{1}^{\prime}, \ldots, \mathbf{p}_{N}^{\prime} ; 0\right)$ :

$$
\left\langle A\left(\mathbf{p}_{1}, \ldots, \mathbf{p}_{N}\right)\right\rangle=\frac{\left(\prod_{j=1}^{N} \int d \mathbf{p}_{j}\right) A\left(\mathbf{p}_{1}, \ldots, \mathbf{p}_{N}\right) \mathcal{K}\left(\mathbf{p}_{1}, \ldots, \mathbf{p}_{N} ; \beta \mid \mathbf{p}_{1}, \ldots, \mathbf{p}_{N} ; 0\right)}{\left(\prod_{j=1}^{N} \int d \mathbf{p}_{j}\right) \mathcal{K}\left(\mathbf{p}_{1}, \ldots, \mathbf{p}_{N} ; \beta \mid \mathbf{p}_{1}, \ldots, \mathbf{p}_{N} ; 0\right)} .
$$

The calculation of the momentum distribution, analogous to the calculation of the spatial density distribution $n(\mathbf{r})$ in [7], can be summarized as follows. First, the propagator in momentum space is evaluated by a Fourier transform of the corresponding propagator in position space obtained in [7]. This results in

$$
\mathcal{K}\left(\mathbf{p}_{1}, \ldots, \mathbf{p}_{N} ; \beta \mid \mathbf{p}_{1}, \ldots, \mathbf{p}_{N} ; 0\right)=\frac{1}{N !} \sum_{\text {permutations } P} \mathcal{K}_{\mathrm{d}}\left(\mathbf{p}_{1}, \ldots, \mathbf{p}_{N} ; \beta \mid P \mathbf{p}_{1}, \ldots, P \mathbf{p}_{N} ; 0\right),
$$

with

$$
\begin{aligned}
\mathcal{K}_{d}\left(\mathbf{p}_{1}, \ldots, \mathbf{p}_{N} ; \beta \mid P \mathbf{p}_{1}, \ldots, P \mathbf{p}_{N} ; 0\right)= & \sqrt{\frac{w_{z} \sinh \left(\beta \hbar w_{z}\right)}{\Omega_{z} \sinh \left(\beta \hbar \Omega_{z}\right)} \frac{w_{x y}^{2} \sinh ^{2}\left(\beta \hbar w_{x y}\right)}{\Omega_{x y}^{2} \sinh ^{2}\left(\beta \hbar \Omega_{x y}\right)}} \prod_{j=1}^{N} K_{d}\left(\mathbf{p}_{j} ; \beta \mid P \mathbf{p}_{j} ; 0\right) \\
& \times \exp \left\{-\frac{P_{z}^{2}}{\hbar m N}\left(\frac{\tanh \left(\beta \hbar \Omega_{z} / 2\right)}{\Omega_{z}}-\frac{\tanh \left(\beta \hbar w_{z} / 2\right)}{w_{z}}\right)\right. \\
& \left.-\frac{P_{x}^{2}+P_{y}^{2}}{\hbar m N}\left(\frac{\tanh \left(\beta \hbar \Omega_{x y} / 2\right)}{\Omega_{x y}}-\frac{\tanh \left(\beta \hbar w_{x y} / 2\right)}{w_{x y}}\right)\right\},
\end{aligned}
$$

where $\mathbf{P}=\sum_{j=1}^{N} \mathbf{p}_{j}$ is the total momentum and $K_{d}\left(\mathbf{p}^{\prime \prime} ; \beta \mid \mathbf{p}^{\prime} ; 0\right)$ is the one-particle propagator in momentum space for a particle in an anisotropic harmonic potential. Next, the sum over permutations appearing in the expression (4) for the mean value $\left\langle\sum_{j=1}^{N} e^{\left.-i \mathbf{q} \cdot \mathbf{p}_{j}\right\rangle}\right.$ is transformed into a cyclic sum (with $M_{\ell}$ the number of cycles of length $\ell$ ). The cyclic summation can be performed for the generating function

$$
g_{1}(u)=\sum_{N=1}^{\infty}\left\langle\sum_{j=1}^{N} e^{-i \mathbf{q} \cdot \mathbf{p}_{j}}\right\rangle u^{N} Z(N),
$$

where $Z(N)=\left(\Pi_{j=1}^{N} \int d \mathbf{p}_{j}\right) \mathcal{K}\left(\mathbf{p}_{1}, \ldots, \mathbf{p}_{N} ; \beta \mid \mathbf{p}_{1}, \ldots, \mathbf{p}_{N} ; 0\right)$ is the partition sum. The result of the cyclic summation is

$$
\begin{aligned}
g_{1}(u)= & \left(\sum_{n=1}^{\infty} u^{n} Z(n)\right) \exp \left\{-\frac{\hbar m q_{z}^{2}}{4 N}\left(\frac{\Omega_{z}}{\operatorname{coth}\left(\beta \hbar \Omega_{z} / 2\right)}-\frac{w_{z}}{\operatorname{coth}\left(\beta \hbar w_{z} / 2\right)}\right)-\frac{\hbar m\left(q_{x}^{2}+q_{y}^{2}\right)}{4 N}\left(\frac{\Omega_{x y}}{\operatorname{coth}\left(\beta \hbar \Omega_{x y} / 2\right)}-\frac{w_{x y}}{\operatorname{coth}\left(\beta \hbar w_{x y} / 2\right)}\right)\right\} \\
& \times \sum_{\ell=1}^{\infty} \frac{u^{\prime} \exp \left\{-\frac{\hbar m}{4}\left[q_{z}^{2} w_{z} \operatorname{coth}\left(\ell \beta \hbar w_{z} / 2\right)+\left(q_{x}^{2}+q_{x}^{2}\right) w_{z} \operatorname{coth}\left(\ell \beta \hbar w_{z} / 2\right)\right]\right\}}{8 \sinh \left(\ell \beta \hbar w_{z} / 2\right) \sinh ^{2}\left(\ell \beta \hbar w_{x y} / 2\right)} .
\end{aligned}
$$

The expectation value $\left\langle\sum_{j=1}^{N} e^{-i \mathbf{q} \cdot \mathbf{p}_{j}}\right\rangle$ for $N$ bosons can then be derived in closed form from the generating function $g_{1}(u)$ by collecting all the terms with the same power in $u$ :

$$
\begin{aligned}
\left\langle\sum_{j=1}^{N} e^{-i \mathbf{q} \cdot \mathbf{p}_{j}}\right\rangle= & \exp \left\{-\frac{\hbar m q_{z}^{2}}{4 N}\left(\frac{\Omega_{z}}{\operatorname{coth}\left(\beta \hbar \Omega_{z} / 2\right)}-\frac{w_{z}}{\operatorname{coth}\left(\beta \hbar w_{z} / 2\right)}\right)-\frac{\hbar m\left(q_{x}^{2}+q_{y}^{2}\right)}{4 N}\left(\frac{\Omega_{x y}}{\operatorname{coth}\left(\beta \hbar \Omega_{x y} / 2\right)}-\frac{w_{x y}}{\operatorname{coth}\left(\beta \hbar w_{x y} / 2\right)}\right)\right\} \\
& \times \sum_{\ell=1}^{N} \frac{Z(N-\ell)}{Z(N)} \frac{\exp \left\{-\frac{\hbar m}{4}\left[q_{z}^{2} w_{z} \operatorname{coth}\left(\ell \beta \hbar w_{z} / 2\right)+\left(q_{x}^{2}+q_{x}^{2}\right) w_{z} \operatorname{coth}\left(\ell \beta \hbar w_{z} / 2\right)\right]\right\}}{8 \sinh \left(\ell \beta \hbar w_{z} / 2\right) \sinh ^{2}\left(\ell \beta \hbar w_{x y} / 2\right)} .
\end{aligned}
$$

Finally, the momentum distribution (2) is found as a Fourier transform of the expectation value (9). The result is 


$$
n_{\text {Bose }}(\mathbf{p})=\left(\frac{1}{4 \pi \hbar m}\right)^{3 / 2} \sum_{\ell=1}^{N} \frac{Z(N-\ell)}{Z(N) \sinh ^{2}\left(\frac{\beta \hbar \Omega_{x y} \ell}{2}\right) \sinh \left(\frac{\beta \hbar \Omega_{z} \ell}{2}\right)} \frac{\exp \left(-\frac{\left(p_{x}^{2}+p_{y}^{2}\right)}{\hbar m A /\left(\Omega_{x y}, w_{x y}\right)}-\frac{p_{z}^{2}}{\hbar m A \lambda\left(\Omega_{z}, w_{z}\right)}\right)}{A \nearrow\left(\Omega_{x y}, w_{x y}\right) \sqrt{A \nearrow\left(\Omega_{z}, w_{z}\right)}},
$$

with

$$
\begin{aligned}
A_{\ell}(\Omega, w)= & \frac{w}{\tanh (\beta \hbar w \ell / 2)} \\
& +\frac{1}{N}\left[\frac{\Omega}{\tanh (\beta \hbar \Omega / 2)}-\frac{w}{\tanh (\beta \hbar w / 2)}\right] .
\end{aligned}
$$

In Fig. 1 this momentum distribution is shown at different temperatures for an isotropically confined Bose gas with $w$ $=\Omega$ and consisting of 1000 bosons. The momentum distribution for $w / \Omega \neq 1$ is qualitatively similar to the distribution with $w / \Omega=1$ shown in Fig. 1 . In the inset, the value of the momentum distribution in the origin is shown as a function of the temperature. As the temperature is lowered below the condensation temperature, the momentum distribution becomes more pronounced in the origin: The average number of bosons with momentum zero rises sharply.

\section{APPROXIMATE VELOCITY DISTRIBUTIONS}

The momentum distribution (10) calculated in the preceding section for a gas of parabolically confined bosons can be related to the Maxwell distribution through a series of approximations. First, in the case of a noninteracting ( $\omega$ $\rightarrow 0$ ), parabolically confined boson gas the momentum distribution (10) becomes

$$
n_{\text {Bose }}^{\text {ideal }}(\mathbf{p})=\left(\frac{1}{4 \pi \hbar m}\right)^{3 / 2} \sum_{\ell=1}^{N} \frac{Z(N-\ell) \exp \left\{-\frac{\left(p_{x}^{2}+p_{y}^{2}\right)}{\hbar m \Omega_{x y} \operatorname{coth}\left(\beta \hbar \Omega_{x y} \ell / 2\right)}-\frac{p_{z}^{2}}{\hbar m \Omega_{z} \operatorname{coth}\left(\beta \hbar \Omega_{z} \ell / 2\right)}\right\}}{Z(N) \Omega_{x y} \sinh \left(\beta \hbar \Omega_{x y} \ell\right) \sqrt{\Omega_{z} \sinh \left(\beta \hbar \Omega_{z} \ell\right)}} .
$$

Furthermore, according to Feynman [9], the MaxwellBoltzmann statistics can be recovered by considering only the cycles of length $\ell=1$ in the cyclic summations of Eq. (10). This means that permutations involving cycles of length larger than 1 play no role any more. Hence the momentum distribution function for distinguishable noninteracting particles in a parabolic potential is

$$
\begin{aligned}
n_{\text {dist }}^{\text {ideal }}(\mathbf{p})= & \left(\frac{\tanh ^{2}\left(\beta \hbar \Omega_{x y} / 2\right) \tanh \left(\beta \hbar \Omega_{z} / 2\right)}{(\pi \hbar m)^{3} \Omega_{x y}^{2} \Omega_{z}}\right)^{1 / 2} \\
& \times \exp \left\{-\frac{\left(p_{x}^{2}+p_{y}^{2}\right)}{\hbar m \Omega_{x y} \operatorname{coth}\left(\beta \hbar \Omega_{x y} / 2\right)}\right. \\
& \left.-\frac{p_{z}^{2}}{\hbar m \Omega_{z} \operatorname{coth}\left(\beta \hbar \Omega_{z} / 2\right)}\right\} .
\end{aligned}
$$

If, moreover, the parabolic confinement were neglected, which corresponds to $\hbar \Omega_{x y}, \hbar \Omega_{z} \ll 1 / \beta$ in Eq. (13), one finally would be left with the Maxwell-Boltzmann momentum distribution for free particles

$$
n_{\text {Maxwell }}(\mathbf{p})=\left(\frac{\beta}{4 \pi m}\right)^{3 / 2} \exp \left\{-\beta \frac{p_{x}^{2}+p_{y}^{2}+p_{z}^{2}}{2 m}\right\} .
$$

In experiments on Bose-Einstein condensed atomic vapors (e.g., [4]), the temperature of a cloud of parabolically trapped bosons was estimated by fitting the Maxwell-
Boltzmann distribution (14) to the tail of the experimentally measured momentum distribution $n_{\text {measured }}(\mathbf{p})$. This can be done by first choosing a sufficiently large threshold momentum $p_{c}$ such that atoms with a momentum larger than $p_{c}$ do not belong to the Bose-Einstein condensate and then minimizing $\int_{p>p_{c}}\left[n_{\text {Maxwell }}(\mathbf{p})-n_{\text {measured }}(\mathbf{p})\right]^{2} d \mathbf{p}$ with respect to the temperature $T$ appearing in the Maxwell-Boltzmann distribution. The temperature obtained by this minimization will be denoted as $T_{\mathrm{MB}}$ and the procedure will be referred to as the "Maxwell-Boltzmann fit procedure."

Since the Maxwell-Boltzmann momentum distribution is a rather crude approximation to the momentum distribution (12) of a gas of noninteracting, parabolically trapped bosons, we rather propose to determine the temperature by fitting $n_{\text {experiment }}(\mathbf{p})$ to the momentum distribution (12) derived in the present paper. This is done by minimizing $\int_{\text {all }} \mathbf{p}\left[n_{\text {Bose }}^{\text {ideal }}(\mathbf{p})-n_{\text {measured }}(\mathbf{p})\right]^{2} d \mathbf{p}$ with respect to the temperature $T$ appearing in the momentum distribution $n_{\text {Bose }}^{\text {ideal }}(\mathbf{p})$ for noninteracting, parabolically trapped bosons. We will denote this procedure as the "Bose-Einstein fit procedure" and the resulting temperature as $T_{\mathrm{BE}}$.

In order to compare the Maxwell-Boltzmann fit procedure with the Bose-Einstein fit procedure, we applied the former on a momentum distribution simulated with $n_{\text {Bose }}^{\text {ideal }}(\mathbf{p})$ $\left[\approx n_{\text {measured }}(\mathbf{p})\right.$ for weakly interacting bosons $]$, at a given temperature $T_{\mathrm{BE}}$. That is, $\int_{p>p_{c}}\left[n_{\text {Maxwell }}\left(\mathbf{p}, T_{\mathrm{MB}}\right)\right.$ $\left.-n_{\mathrm{Bose}}^{\text {ideal }}\left(\mathbf{p}, T_{\mathrm{BE}}\right)\right]^{2} d \mathbf{p}$ was minimized with respect to $T_{\mathrm{MB}}$, keeping $T_{\mathrm{BE}}$ and $p_{c}$ fixed. 


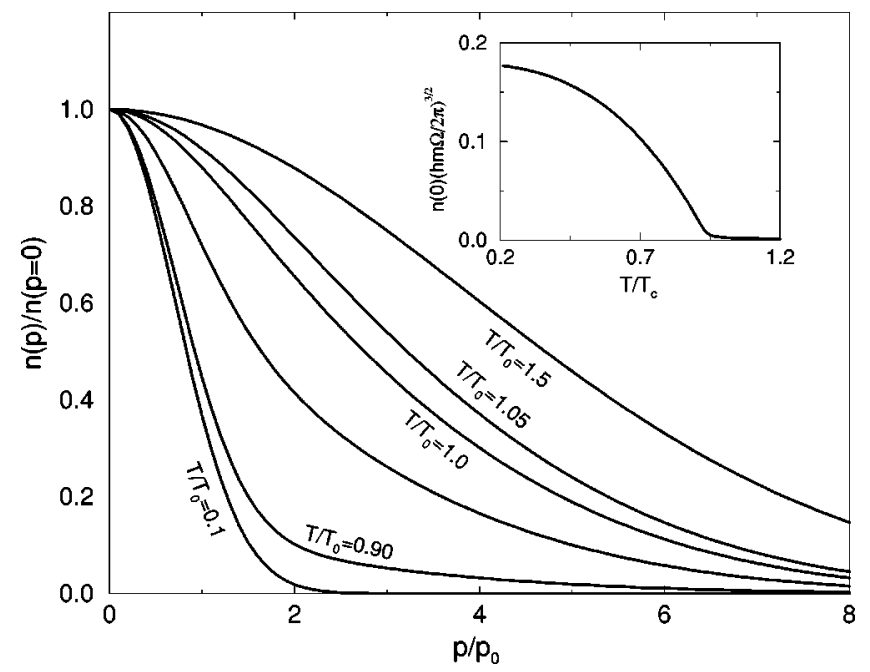

FIG. 1. Average number $n(\mathbf{p}) / n(\mathbf{p}=0)$ of particles with a momentum p, shown at several temperatures for a Bose gas in an isotropic parabolic confinement, as a function of the momentum. Units are chosen such that $\hbar=m=\Omega=1$. In this set of units, the momentum is expressed in $p_{0}=\sqrt{\hbar m \Omega}$. In the inset, the momentum distribution at $\mathbf{p}=\mathbf{0}$ is shown as a function of temperature, with $T_{0}=\hbar \Omega / k_{B}[N / \zeta(3)]^{1 / 3}$. For this figure we have chosen $w=\Omega$. The momentum distribution for $w / \Omega \neq 1$ is qualitatively similar to the distribution for $w / \Omega=1$.

The result $T_{\mathrm{MB}}$ of the minimization is compared to $T_{\mathrm{BE}}$ in Fig. 2, where the ratio $\left(T_{\mathrm{BE}}-T_{\mathrm{MB}}\right) / T_{\mathrm{BE}}$ is shown as a function of $T_{\mathrm{BE}}$ at a fixed threshold momentum $p_{c}=5 \sqrt{\hbar m w}$. In the Bose-Einstein condensed phase the discrepancy between $T_{\mathrm{BE}}$ and $T_{\mathrm{MB}}$ increases when the number of bosons increases, whereas for $T_{\mathrm{BE}}>T_{0}=\hbar w[N / \zeta(3)]^{1 / 3}$, the discrepancy decreases when the number of bosons is augmented, as shown in the inset of Fig. 2. For temperatures well above the condensation temperature $T_{0}$, the ratio $\left(T_{\mathrm{BE}}-T_{\mathrm{MB}}\right) / T_{\mathrm{BE}}$ is small and the Maxwell-Boltzmann fit procedure gives a reliable temperature estimate in this high-temperature regime. The difference between $T_{\mathrm{BE}}$ and $T_{\mathrm{MB}}$ becomes appreciable below the condensation temperature $T_{0}$. The present analysis therefore shows that $\left(T_{\mathrm{BE}}-T_{\mathrm{MB}}\right) / T_{\mathrm{BE}}$ is not negligible in the Bose-Einstein condensed phase and that the BoseEinstein fit procedure is more appropriate than the MaxwellBoltzmann fit procedure for the determination of the temperature.

\section{CONCLUSION}

In this paper we calculated the momentum distribution function (10) for a Bose gas in an anisotropic parabolic trapping potential, including quadratic interparticle interactions. In the absence of interparticle interactions, this momentum distribution simplifies to the momentum distribution function (12) of an ideal boson gas in a harmonic confining potential. The Maxwell-Boltzmann distribution (14) follows from Eq. (12) by the further simplifications of neglecting the boson statistics and the parabolic confinement. The underlying as-

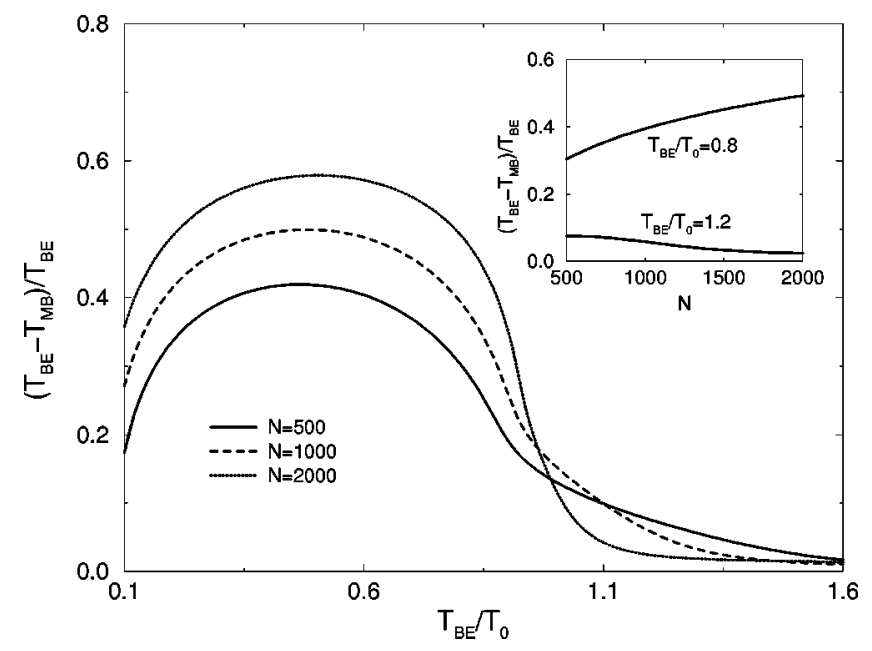

FIG. 2. Relative difference between the temperature $T_{\mathrm{MB}}$ found by fitting a Maxwell-Boltzmann distribution and the actual temperature $T_{\mathrm{BE}}$, representing $\left(T_{\mathrm{BE}}-T_{\mathrm{MB}}\right) / T_{\mathrm{BE}}$ versus $T_{\mathrm{BE}}$ in units $T_{0}$ $=\left(\hbar \Omega / k_{B}\right)[N / \zeta(3)]^{1 / 3}$. This is shown for 500,1000, and 2000 noninteracting bosons. In the inset, the dependence of $\left(T_{\mathrm{BE}}\right.$ $\left.-T_{\mathrm{MB}}\right) / T_{\mathrm{BE}}$ on the number of particles is shown.

sumption in these derivations is that the number of bosons is sufficiently large to maintain the temperature concept from the thermodynamical limit. Assuming furthermore thermodynamical equilibrium, two temperature estimates were compared. Since the temperature estimates used in experiments on Bose-Einstein condensed atomic vapors (e.g., [4]) are obtained by fitting the Maxwell-Boltzmann distribution (14) to the tail of the experimentally measured momentum distribution $n_{\text {measured }}(\mathbf{p})$, we derived the corresponding temperature $T_{\mathrm{MB}}$ by fitting the large-momentum tail of the MaxwellBoltzmann distribution to the large-momentum tail of the momentum distribution of the Bose gas (12). This temperature estimate was compared to the temperature $T_{\mathrm{BE}}$ in the momentum distribution of the boson gas. We find that $T_{\mathrm{MB}}$ substantially deviates from the correct temperature $T_{\mathrm{BE}}$ in the Bose-Einstein condensed phase and hence that the BoseEinstein fit procedure is more appropriate than the MaxwellBoltzmann fit procedure for the determination of the temperature.

\section{ACKNOWLEDGMENTS}

Part of this work was performed in the framework of the FWO under Projects Nos. 1.5.729.94, 1.5.545.98, G.0287.95, G.0071.98, and WO.073.94N (Wetenschappelijke Onderzoeksgemeenschap over "Laagdimensionele Systemen," Scientific Research Community of the FWO on "Low Dimensional Systems"), the "Interuniversitaire Attractiepolen-Belgische Staat, Diensten van de Eerste Minister-Wetenschappelijke, Technische en Culturele aangelegenheden," and in the framework of the BOF NOI projects of the Universiteit Antwerpen. J.T. and F.B. acknowledge the FWO for financial support. 
[1] M. H. Anderson, J. R. Ensher, M. R. Matthews, C. E. Wieman, and E. A. Cornell, Science 269, 198 (1995).

[2] K. B. Davis, M.-O. Mewes, M. R. Andrews, N. J. Van Druten, D. S. Durfee, D. M. Kurn, and W. Ketterle, Phys. Rev. Lett. 75, 3969 (1995).

[3] C. C. Bradley, C. A. Sackett, J. J. Tollett, and R. G. Hulet, Phys. Rev. Lett. 75, 1687 (1995).

[4] J. R. Ensher, D. S. Jin, M. R. Matthews, C. E. Wieman, and E. A. Cornell, Phys. Rev. Lett. 77, 4984 (1996).

[5] L. F. Lemmens, F. Brosens, and J. T. Devreese, Phys. Rev. E
53, 4467 (1996).

[6] F. Brosens, J. T. Devreese, and L. F. Lemmens, Phys. Rev. E 55, 227 (1997).

[7] F. Brosens, J. T. Devreese, and L. F. Lemmens, Phys. Rev. E 55, 6795 (1997).

[8] J. Tempere, F. Brosens, J. T. Devreese, and L. F. Lemmens, Solid State Commun. 107, 51 (1998).

[9] R. P. Feynman, Statistical Mechanics (Addison-Wesley, Reading, MA, 1972). 